\title{
Vaginal Birth after Cesarean Delivery
}

National Cancer Institute

\section{Source}

National Cancer Institute. Vaginal Birth after Cesarean Delivery. NCI Thesaurus. Code C81302.

A vaginal birth in a woman with one or more previous cesarean births. (reVITALize) 